\title{
Performance Improvement of Channel Quality Adaptive protocol for effective routing in VANET
}

\author{
Dhanashree Tajne \\ PG Student \\ Electronis and Telecommunication Department \\ G. H. Raisoni College of Engineering
}

\author{
Dharamveer Choudhari \\ Assistant Professor \\ Electronics and Telecommunication \\ Department \\ G. H. Raisoni College of Engineering
}

\begin{abstract}
Many applications are built in broadcast communication and thus efficient routing methods are critical. In this paper, DADCQ protocol is addressed. The advantage of this protocol is that it uses distance method to select the forwarding nodes in order to forward the data packets. This method depends completely on the decision threshold. In order to calculate decision threshold, node density, spatial distribution pattern and wireless channel quality is used. Broadcast protocols should be adaptive to all the changes in the network. They should also be adaptive to the all variations in the parameters. The proposed paper facilitates the impact analysis of distance dependent DSRC fading channel.
\end{abstract}

\section{General Terms}

VANET, RSU, DSRC, Spatial Distribution Pattern

\section{Keywords}

DADCQ, distance method, Rician fading factor, distance threshold

\section{INTRODUCTION}

VANET is a special type of mobile ad-hoc networks where vehicles are simulated as mobile nodes. VANET contains of two types of entities: access points and vehicles. Access points are generally fixed and connected to the internet and they could participate in the vehicular distribution pattern. The growth of increased number of vehicles is well equipped with wireless transceivers in order to communicate with each other. To enhance the safety of drivers and provide the comfortable driving environment, messages with several information need to be sent over the network and this is termed as inter-vehicular communication. There are three types of routing: unicast routing, multicast routing and broadcast routing. In unicast routing, communication takes place only in two dedicated vehicles viz source and destination. In multicast routing, only one source transmits information to several nodes at same instance. In broadcast routing, source sends information to many nodes in its vicinity and rebroadcasts a message if not delivered to any of the node.

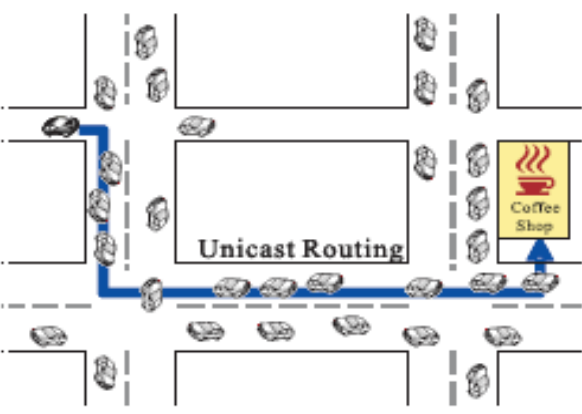

Fig. (a)

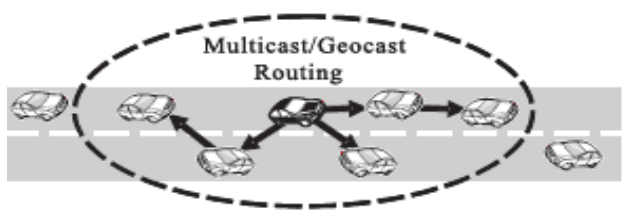

Fig. (b)

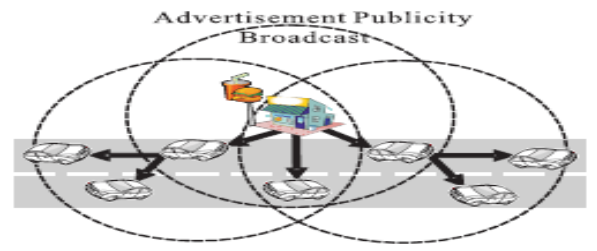

Fig. (c)

Fig. (a) shows unicast routing, fig.(b) shows multicast routing and fig. (c) shows broadcast routing. VANET also have various special characteristics that distinguish it from various mobile ad-hoc networks. Some of those special characteristics are high mobility, self-organization, distribution communication, no restriction of network size, road pattern restrictions etc.

Emerging classes of vehicular network utilizes broadcast as a mechanism which transfers data packets. The distribution of nodes is highly variable in VANET. Network densities can vary from one or two neighbors to a wide number of 
neighbors. Dedicated Short Range Communication (DSRC), layer 1 and 2 based on IEEE 802.11 designed vehicle-tovehicle and vehicle-to-infrastructure, allows transmission ranges up to $1000 \mathrm{~m}$ [1]. The distribution pattern, independent of density, can also vary. In isolated highways, nodes are along one dimensional path. In urban area, nodes appear in two dimensional paths and road crossings and suburban area exhibit several combinations in between. Another problem with these wireless communication network is the loss of packets as they traverse the medium. Multipath fading occurs as signal splits into several signals due to being reflected off objects in the environment. Packets can also be lost when different transmitted signals interfere each other. This phenomenon is called collisions. The following simulation gives a random urban scenario.

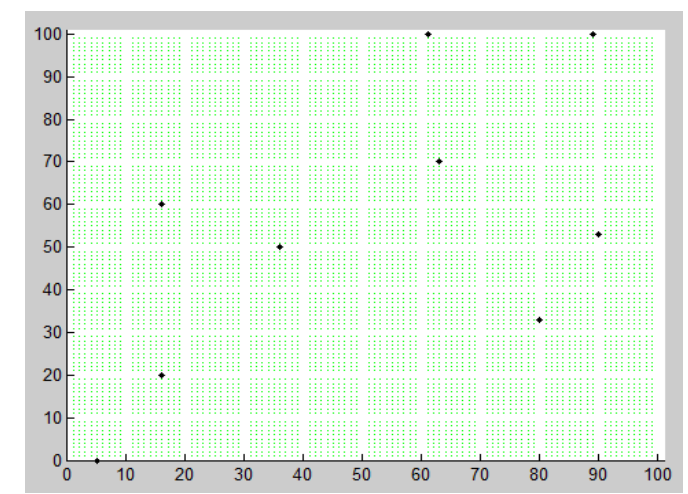

\section{DESIGNING OF BROADCAST PROTOCOL}

The contribution to this work is as summarized below.

DADCQ protocol: This protocol combine's local distribution pattern with other factors like distance method to select nodes for forwarding data packets. Previous broadcast protocols proposed for ad-hoc networks which use distance method for selection of rebroadcasting nodes is less comprehensive supplemental information. But this protocol gives more information than any other broadcast protocol proposed earlier. This extra information is used to make protocol adaptive more than protocols which were proposed earlier.

Quadrat method for Spatial Distribution function: One of the important factors that help to design the rebroadcasting threshold function is the local node density pattern. Quadrat method is used to characterize the spatial distribution of nodes [14]. Distribution pattern may affect the behavior of many broadcast protocols. This contribution is applicable in a wider context.

\section{DISTANCE METHOD}

The proposed DADCQ protocol utilizes distance method in order to select the forwarding nodes. The distance method uses minimum distance between the sender and the destination which is desired to be one hop distance[20]. The method appeals to the intuition that if the node is situated near the sender node then the message is completely delivered and there is no need of rebroadcasting the message. Rebroadcasting a message is expected always whenever the node is located at a large distance from the sender node [12]. The complete algorithm is given as follows.
1. Initialize $\mathrm{D}=1$

2. When message is received set $\mathrm{d}$ to the distance to the sender, $\mathrm{D}=\min \{\mathrm{D}, \mathrm{d} / \mathrm{r}\}$, and set a random backoff timer.

3. If a message is received during backoff then repeat the above process.

4. When the backoff expires, rebroadcast if $\mathrm{D}<\mathrm{D}_{\mathrm{c}}$.

If the value of $D_{c}$ is too high, reachability will be degraded. If the value is set too low then many nodes will be prevented from rebroadcasting. The value of $D_{c}$ should be decided in such a way that it should be adjustable to the varying node densities [3]. At low node densities, $\mathrm{D}_{\mathrm{c}}$ must be lower to allow the message to get propagated to the entire network. When the node density is higher, we can set $D_{c}$ a little aggressive in order to eliminate excess transmissions.

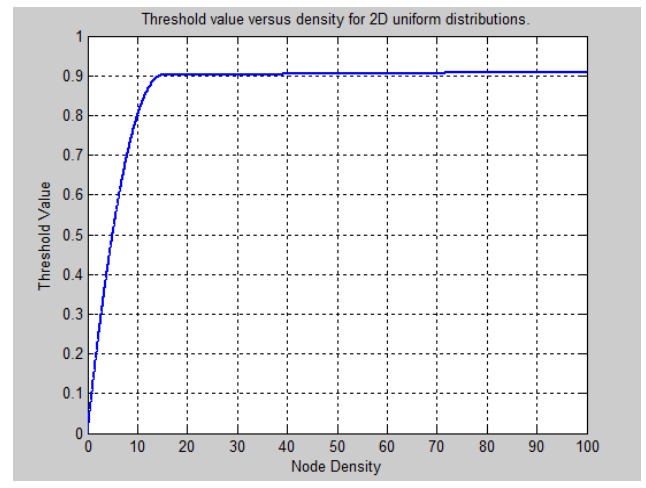

The above figure gives the plot of threshold value versus node density. As shown in the above figure the threshold should be less aggressive as the node density is confined to the one dimensional path. Since the $2 \mathrm{D}$ threshold curve is more aggressive, if it is used in linearly oriented networks, reachability will not meet the required target. In Quadrat method, a quadrat metric is defined which is the ratio of variance to the mean.

$$
\mathrm{Q}=\frac{V(n)}{E(n)}
$$

Different values of $\mathrm{Q}$ decide node distribution. If $\mathrm{Q}=1$ then variance is equal to the mean and the distribution is uniformly random. If $\mathrm{Q}>1$ then the nodes are clustered together as the variance become larger than the mean. If $Q<1$ then variance of frequencies is less than the mean, indicating the nodes are more evenly spaced than expected in uniformly distributed networks.

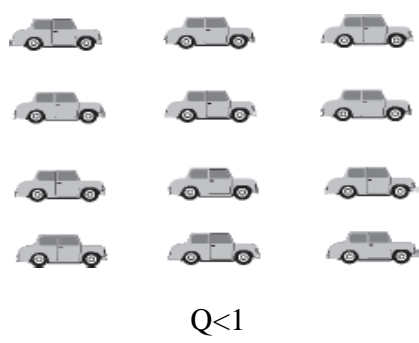

As shown in above figure, the value of $\mathrm{Q}$ is less than 1 as a result of which nodes are equally distributed. 


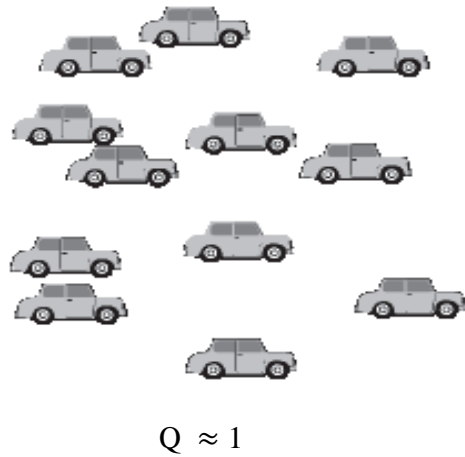

In above figure, vehicular nodes are clustered together because the value of $\mathrm{Q}$ is approximately equal to 1 .

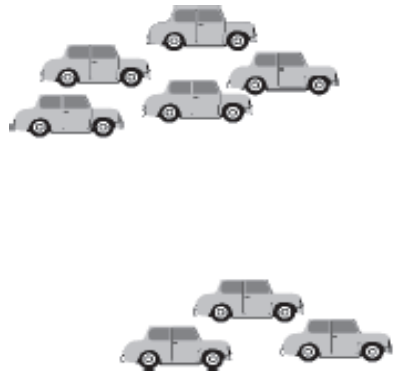

$\mathrm{Q}>1$

Fig: Figure illustrating several values of $\mathbf{Q}$

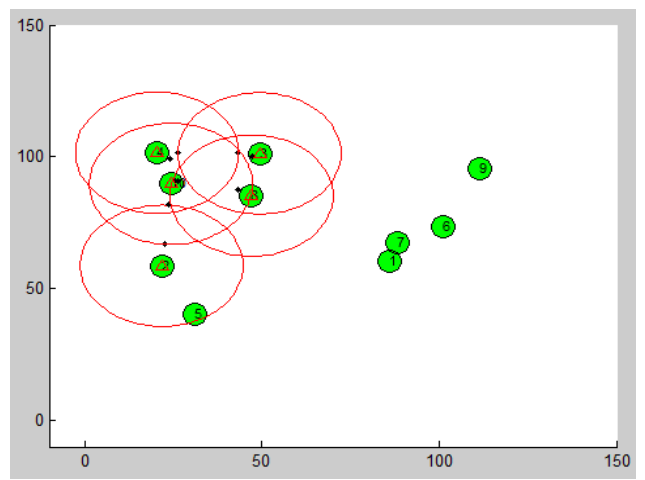

Fig: Simulation results illustrating communication between several nodes

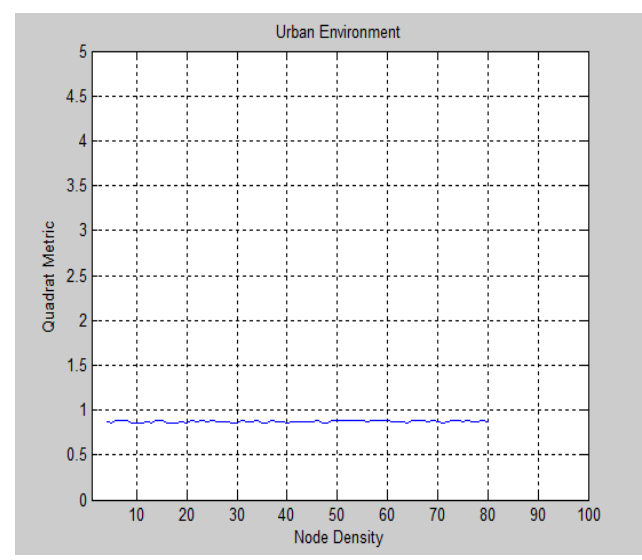

Fig: Plot of Quadrat metric and Node density

\section{ADAPTATION TO CHANNEL QUALITY}

In this paper an assumption is made that the wireless channel and the medium access layer protocol deliver messages between nodes located within the transmission range of each other. This gives perfect reliability. When two nodes send messages at a time then it might happen that those two messages interfere each other and one or both messages may be lost at the destination. It is called fading. In other words, fading is a phenomenon where multiple parts of a signal travelling along different paths interfere each other, degrades communication even when only single node is transmitting. Multihop wireless broadcast protocols must be able to operate effectively in all such circumstances.

\subsection{Modeling Fading}

In VANET, fading may be modeled as Rician fading. In Rician fading, the received signal is assumed a strong component of line-of-sight among scattered multipath components. As the vehicles are moving continuously, these movements can cause temporary disruptions in line-of-sight communications. These disruptions cannot be captured by such standard models. The Rician model allows the fading intensity to be easily quantified. The parameter, K, easily quantifies the relative strength of the line-of-sight signal in Rician fading.

\subsection{Designing A Threshold Function Using \\ $\mathbf{K}$}

In this section, formulae are given which can be used to find out the threshold function. The following expressions make use of node density $(\mathrm{N})$, quadrat metric $(\mathrm{Q})$ and Rician fading factor $(\mathrm{K})$. Using these variables $\alpha$ and $\beta$ are calculated along with the threshold function. On the basis of the literature survey done earlier, it is observed that $\beta$ in $2 \mathrm{D}$ scenario is roughly thrice than that in $1 \mathrm{D}$ scenario. The following are the expressions for calculating $\alpha, \beta$ and $D_{\max }$.

$$
\begin{aligned}
& \alpha=-0.200\left(\frac{2(Q-1)}{0.125 N}+1\right)^{-1} \\
& \beta=6.25 e^{-0.1 K}\left(\frac{2(Q-1)}{0.125 N}+1\right)^{-1} \\
& D_{\max }=0.75-0.237 e^{-0.1 K}
\end{aligned}
$$

$\mathrm{N}, \mathrm{Q}$ and $\mathrm{K}$ are measured independently for each node.

\subsection{Finding $K$}

The proposed protocol calculates the rebroadcasting threshold as a function of N, Q and K. N and Q can be easily calculated on the basis of messages which are being sent. The calculation of $\mathrm{K}$ is not as easy as the calculation of $\mathrm{N}$ and $\mathrm{Q}$. The value of $\mathrm{K}$ should be such that it should not be too less or too large [7]. If the value of $\mathrm{K}$ is chosen much higher than that of the minimum value then some methods may result in several rebroadcasts. This will in turn result in inefficient use of bandwidth. Although. Nodes probably cannot accurately measure $\mathrm{K}$ dynamically, they may be able to use measured node distribution to provide some feedback to the value of $\mathrm{K}$ 
The values of $\alpha, \beta$ and $D_{\max }$ for $\mathrm{N}=10$ are given as.

\begin{tabular}{|c|c|c|c|}
\hline $\mathrm{K}$ & $\alpha$ & $\beta$ & $\mathrm{D}_{\max }$ \\
\hline 0 & 0.0066 & -0.2076 & 0.5130 \\
\hline 5 & 0.0137 & -0.2605 & 0.6063 \\
\hline 10 & 0.0026 & -0.0300 & 0.6628 \\
\hline 15 & 0.0014 & -0.0100 & 0.6971 \\
\hline 20 & 0.0022 & -0.0092 & 0.7179 \\
\hline 25 & 0.0019 & -0.0048 & 0.7305 \\
\hline
\end{tabular}

\section{OVERHEAD BEACONING}

Beaconing is a process in which every node sends a message after every $T_{b}$ sec to all its neighbors. That message is called a beacon. These beacons will never be retransmitted. Nodes send beacons at a fixed rate. So in any span of $T_{b}$ sec, every node in the network will send exactly 1 beacon. Timing of beaconing is not synchronized at any instance; the only thing common in all the nodes is the duration of $T_{b}$ sec. Individual nodes set a timer of $T_{b}$ sec and at the end of that timer nodes compile their messages and send them again. The information contained in message completely depends on the propagation algorithm. DADCQ calculates $\mathrm{N}$ and $\mathrm{Q}$ so that nodes get acquainted with their current location. $\mathrm{N}$ is calculated on the basis of number of beacons received from the neighbors. Q is calculated on the basis of the location present in each beacon. DADCQ has 12 bytes of beacon. If the network is static, the beacon period of $T_{b}$ sec is longer with high accuracy of data. On the contrary, for mobile nodes beacon period reduces, lowering the accuracy of the message.

\section{ATTACKS AFFECTING WOKING IN VANET}

VANET is facing many attacks. Some of them are described as follows.

\subsection{Denial Of Service Attacks}

DOS attacks are a type of attack which are caused by the network insiders and outsiders [14] and provide the network which is not available to the real users. This is done by flooding the control channel with high amount of naturally generated messages and thus stopping the connection. This results in improper functioning of OBU and RSU.

\subsection{Sybil Attack}

This attack forges the identity of many vehicles and these identities are used to cast any type of attack on the system [15]. These identities then create an illusion that there is traffic on the road.

\subsection{Message Suppression Attack}

In this type of attack, the attacker selectively drops the message packets. These packets may hold critical information for the receiver. The attacker suppresses these packets. These suppressed packets can then be used again whenever required. The goal of this attack is to prevent insurance authorities from learning about the vehicular collisions [16].

\section{CONCLUSION}

VANETs exhibit a wide variety in node density, spatial distribution and channel quality. So a broadcast protocol should work properly in such variation. DADCQ protocol is for broadcasting in VANET. It uses distance method in order to select the forwarding nodes. This protocol depends on the distance threshold in order to select the forwarding nodes. This protocol analyzes spatial analysis with the help of quadrat method. Spatial analysis is done in order to characterize the distribution pattern at each node. This protocol provides high reachability and efficient use of bandwidth 2D scenario with such varying fading intensity and node density.

\section{REFERENCES}

[1] S.-Y. Ni, Y.-C. Tseng, Y.-S. Chen, and J.-P. Sheu, "The Broadcast Storm Problem in a Mobile Ad Hoc Network," Proc. ACM/IEEE MobiCom, pp. 151162,1999

[2] M.J. Slavik and I. Mahgoub, "Statistical Broadcast Protocol Design for Unreliable Channels in Wireless Ad-Hoc Networks," Proc. IEEE GlobeCom, Dec. 2010.

[3] M.J. Slavik and I. Mahgoub, "Designing Statistical Multi-Hop Wireless Broadcast Protocols Using Confidence Levels from Stochastic Models of Reachability," Proc. Int'l Wireless Comm. And Mobile Computing Conf. (IWCMC '11), 2011

[4] M.J. Slavik and I. Mahgoub, "Adapting Statistical Broadcast to Linearly Oriented Networks for VANETs," Proc. IEEE Int'l Conf. Wireless and Mobile Computing, Networking and Comm. (WiMob '10), Oct. 2010

[5] W. Viriyasitavat, F. Bai, and O. Tonguz, "UVCAST: An Urban Vehicular Broadcast Protocol," Proc. IEEE Vehicular Networking Conf. (VNC), pp. 25-32, Dec. 2010

[6] M. Mariyasagayam, T. Osafune, and M. Lenardi, "Enhanced Multi-Hop Vehicular Broadcast (MHVB) for Active Safety Applications," Proc. Seventh Int'l Conf. ITS Telecomm. (ITST '07), pp. 1-6, June 2007.

[7] J. Paul, J.S. Davis, and M.G. Linnartz, "Vehicle to Vehicle RF Propagation Measurements," Proc. Conf. Record of the 28th Asilomar Conf. Signals, Systems and Computers, vol. 1, pp. $470-474$, Oct. 1994.

[8] J. Cartigny, D. Simplot, and J. Carle, "Stochastic Flooding Broadcast Protocols in Mobile Wireless Networks," technical report, Universite' des Sciences et Technologies de Lille 1, http:// citeseer.ist.psu.edu/525199.html, May 2002.

[9] W. Lou and J. Wu, "Toward Broadcast Reliability in Mobile Ad Hoc Networks with Double Coverage," IEEE Trans. Mobile Computing, vol. 6, no. 2, pp. 148-163, Feb. 2007.

[10] M. Boban, T. Vinhoza, M. Ferreira, J. Barros, and O. Tonguz, "Impact of Vehicles as Obstacles in Vehicular Ad Hoc Networks," IEEE J. Selected Areas in Comm., vol. 29, no. 1, pp. 15-28, Jan. 2011. 
[11] M.J. Slavik and I. Mahgoub, "Spatial Distribution and Channel Quality Adaptive Protocol for Multihop Wireless Broadcast Routing in VANET," Proc. IEEE Transactions on Mobile Computing, VOL. 12, NO. 04, April 2013

[12] R. Thomas, An Introduction to Quadrat Analysis. Geo Abstracts Ltd., 1977.

[13] Sherali Zeadally, Ray Hunt, Yuh-Shyan Chen, Angela Irwin, Aamir Hassan, "Vehicular ad hoc networks (VANETS): status, results, and challenges," Springer Science +Business Media,LLC 2010.

[14] Mina Rahbari and Mohammad Ali Jabreil Jamali, "Efficient Detection of Sybil Attack Based on Cryptography in VANET," IJNSA, Vol.3, No.6, November 2011.

[15] Mushtak Y. Gadkari , Nitin B. Sambre, "VANET: Routing Protocols, Security Issues and Simulation Tools," IOSR Journal of Computer Engineering, July-Aug. 2012 